\title{
BIM SYSTEM FOR THE CONSERVATION AND PRESERVATION OF THE MOSAICS OF SAN MARCO IN VENICE
}

\author{
F. Fassi ${ }^{\text {a }}$, L. Fregonese ${ }^{\text {a }}$, A. Adami ${ }^{\text {a }}$, F. Rechichi ${ }^{\text {a }}$ \\ ${ }^{\text {a }}$ Dept. ABC, Politecnico di Milano, \\ francesco.fassi@polimi.it, luigi.fregonese@polimi.it, andrea.adami@polimi.it, fabrizio.rechichi@polimi.it
}

Commission II, WG VIII

KEY WORDS: BIM, complex 3D model, web sharing, 3D visualization, orthophoto

\begin{abstract}
:
The Basilica of San Marco in Venice is a well-known masterpiece of World Heritage. It is a real multi-faceted architecture. The management of the church and its construction site is very complicated, and requires an efficient system to collect and manage different kinds of data. The BIM approach appeared to be the most suitable to collect multi-source data, to monitor activities and guarantee the well-timed operations inside the church. The purpose of this research was to build a BIM of the Basilica, considering all aspects that characterize it and that require particular care.

Many problems affected the phase of the acquisition of data, and forced the team to establish a clear working pipeline that allowed the survey simultaneously, hand in hand, with all the usual activities of the church. The fundamental principle for the organization of the whole work was the subdivision of the entire complex in smaller parts, which could be managed independently, both in the acquisition and the modelling stage. This subdivision also reflects the method used for the photogrammetric acquisition. The complexity of some elements, as capitals and statues, was acquired with different Level of Detail (LoD) using various photogrammetric acquisitions: from the most general ones to describe the space, to the most detailed one 1:1 scale renderings. In this way, different LoD point clouds correspond to different areas or details.

As evident, this pipeline allows to work in a more efficient way during the survey stage, but it involves more difficulties in the modelling stage. Because of the complexity of the church and the presence of sculptural elements represented by a mesh, from the beginning the problem of the amount of data was evident: it is nonsense to manage all models in a single file.

The challenging aspect of the research job was the precise requirement of the Procuratoria di San Marco: to obtain the 1:1 representation of all the mosaics of the Basilica. This requirement significantly increased the effort in the acquisition stage, because it was necessary to reach a submillimetre resolution in the photographic images sufficient to distinguish perfectly each single tessera, also in the highest domes (28 meters). Furthermore, it introduced a new problem about the management of the gigapixel orthophotos.

The BIM approach presented in this paper tries to offer a solution to all these problems. The BIM application is based not on commercial software, but on a self-implemented system, which was previously tested on the Main Spire of Milano Cathedral. The multi-scale and multi-area approach have also been maintained in the BIM construction phase.

In the case of Basilica di San Marco, the most important requirement was the management of the orthophotos of each single element. It was necessary to give the user the possibility to recover, for each item, not only the geometric model, but also the raster representation -orthophoto- of its surface: in order to do it, the BIM model acts as a three-dimensional catalogue.
\end{abstract}

\section{INTRODUCTION}

\subsection{An ad-hoc BIM system for the Basilica}

The Basilica di San Marco in Venice is a centre of history, art and faith that, for over a thousand years, has attracted millions of visitors driven by devotion and desire to know. As soon as one enters the basilica, the golden mosaics of the vaults immediately attract the visitor's attention and remind of other spaces and churches as Haghia Sophia in Istanbul. Walls and columns, with their marbles, recall the stories of John Ruskin and Marcel Proust.

This inestimable heritage, made not only of mosaics and marbles, but also of the history of capitals and columns brought together from different countries of the Venetian dominion, must be preserved by the effects of time and, at the same time, it is necessary to use all the possible instruments and tools to ensure it a long life. The management of such a complex architecture requires an efficient system, which allows to collect and handle different kinds of data: 2D drawings, 3D representations, historical documents, and analyses. Today the most suitable approach is offered by BIM both to collect different data and to use all those data also in a planning phase, in accordance with the previous interventions, the state of conservation and all the activities which are carried on in the church. Anyway, the BIM for Cultural Heritage does not work only as a repository of documents, but can have many other functions such as representing i) a global model for the extraction of new drawings in an automatic way, ii) the base for the design of the maintenance intervention, iii) a way to optimize the conservation procedures, iv) a mean to integrate graphical and informational data flows, v) a life cycle instrument to make operations more effective, faster and cheaper. All these roles could be taken by the 3D model of the building, which become the core of the information system. It is not a simple task: it must be implemented according to the characteristics of the architecture and the goals of BIM.

In the case of San Marco, the requirements were a 3D model at the scale 1:50 with the possibility to extract many other bidimensional representations at different levels and positions. At the same time, the customer asked to pay particular attention to the appearance of surfaces, as they are interested to use it for 
conservation activities related to the mosaics.

In order to translate these requirements into practical terms, the first demand was for a high-detailed 3D model showing the architecture and decoration and its deformations. Highresolution orthophotos are the most suitable instruments to represent an object and its appearance (Fassi et al., 2017): they are correctly measurable and give information about the state of conservation of surfaces. Finally, in the case of mosaics, an orthophoto gives detailed information about characteristics and position of each single tessera, which can be given, otherwise, with a big effort in terms of drawing and time.

Considering the pros and cons of using commercial or selfimplemented BIM authoring software, the choice for San Marco was to use a system realized on purpose. The choice fell on the WebBIM3D (Fassi et al., 2012), a system that has been implemented for the management of the restoration works of the Main Spire of Milan Cathedral. It is a double-face system developed ad hoc for Cultural Heritage cases of study. It allows combining the modelling tools of a very popular NURBS modelling software -Rhinoceros- with an external database, which is very adaptable to each requirement and a WEB tool that allows the navigation and the reading/writing of data inside the system. It is possible to visualize a single part or the whole model or combination of parts in relation to their position or relation. This fosters the work of the final user who can decide what to visualize according to his needs, thus overcoming the difficulties to navigate inside elaborated 3D models, or the obligation to split them a priori into fix subparts. Moreover, considering the complexity and the dimension of the $3 \mathrm{D}$ models, many expedients have been implemented to allow a correct navigation and management of the model.

\subsection{The challenges}

This project presented many challenges of different nature, starting with the survey phase, going through the elaborations process till the fruition of the data.

After a description of context and requirements, this paper will first of all describe the survey stage, paying attention to all the problems connected to the characteristics of the church itself: dimension, materials properties, illumination and the continuous flow of tourist and workers. The following part will discuss the modelling operations, according to the results of the previous acquisition stage and vindicate the necessities of a structured model for subsequent data management. The third part will contain an in-deepth analysis of the process for the creation of orthophotos and their management inside the specifically implemented BIM system.

\section{MOSAICS AND ORTHOPHOTOS}

The mosaics of Basilica di San Marco underwent many restoration works during the centuries, since the end of the $13^{\text {th }}$ century ${ }^{1}$.

The mosaics of the Basilica belong to different historical periods starting from the $11^{\text {th }}$ century. Today the mosaics are not completely the original ones as they are the results of different interventions such as conservation, replacement, integration and additions carried out in various periods. In this sense, the mosaics of the Basilica have not only an artistic value, but they are a proof of different conservation theories developed along their history.

The interventions dated before the end of the $13^{\text {th }}$ century

1 For a complete survey of the history of mosaics see http://www.basilicasanmarco.it/). showed a high respect of the original mosaic; in fact, only details such as the background were retouched, whereas the original figures were not modified.

During the renaissance, due to a different concept of their work and value, the restorers modified the original concepts according to their own style. Likewise, the workers of the $16^{\text {th }}$ and $17^{\text {th }}$ century not only were not afraid to integrate small parts but renewed entire parts. In the 18th century, there was a new respect for old mosaics and so even a more conservative approach. But, at the same time, there was a negative value judgement on the most recent mosaics.

The big interventions of the $19^{\text {th }}$ century followed the period of abandonment of the church after the downfall of Venice. The restorations, even if for big areas, aimed at reproducing the original drawings. At the beginning of the $20^{\text {th }}$ century, the operations were more sophisticated and refined: the workers removed the surface to be restored ("lievo") and also tried the so called "restoration from behind" which was done by maintaining the mosaic in its original position and operating on the behind masonry.

In the second half of the $20^{\text {th }}$ century, the philosophy of interventions was toward a very rigorous respect for history. More than 3000 square meters were restored by using "the new method": damaged areas were restored; the bad interventions of the past were modified and also new technologies and methods were implemented.

Today the philosophy of intervention is based on a great respect for the history and its development, in a period when there are many risks for the mosaics. The main damages are caused by the detachment from the masonry, the falling off of tesserae, structural alteration and from break-up of the basic layers that ensure adhesion in addition to the atmospheric conditions and humidity. They produced the tesserae crumble and in some cases their fall down in small sections.

The intervention of consolidation is made with by means of a liquid mortar obtained by mixing milk of lime and finely powdered marble and gradually adding as required an acrylic resin. But when damage is due to crumbling of the tessera fixing mortar, the so-named "rovigno", the operation is far more difficult. When the consolidation is not possible, it is necessary to remove the area ("lievo") and clean it, then the replacement of deteriorated base layers with new ones.

According to the values of the mosaics and these restoration practices, it is very important to have a correct documentation of all mosaics that can be used as the base for further analysis or where to record all annotations (deterioration and interventions). Moreover, the very high resolution documentation, in form of orthophoto, can be also printed at scale $1: 1$ to be used as a cartoon to re-create the most deteriorated part of the surface. Following this necessity, the Politecnico of Milano realized in 2003-2005 the orthophoto in scale $1: 1$ of the whole pavement of the Basilica (Fregonese et al, 2006). Of course, these requirements imply that the orthophoto has a very high resolution in order to see the joints between different tessera and with trusty colours.

\section{3D SURVEY FOR THE MODELLING}

The Basilica itself presented some very particular characteristics due to its history and its architecture. This affected the acquisition method (Fregonese et al, 2017). The architecture is rather complex: it is composed by three naves, a transept and many chapels and apses, organized in two levels, with different arches, vaults and domes. In addition the basilica is characterized by the absence of break-lines, that are the sharp edges, which usually build the architecture and are used to 
reconstruct and represent the architecture analogically or digitally, in 2D or in 3D. It means that in many cases, above all in the upper part of the church decorated by mosaics, there are no edges. The architecture seems to be "an object in one piece": it is very difficult or impossible to understand where an object ends and where the other begins. This implies difficulties in the modelling stage, but above all for the orthophotos: the actual solution for orthophoto is to identify an artificial life to divide, for example, the vault from the wall.

The materials of the church are very rich, composed by glass and golden tesserae on the walls and by different kinds of marble on the floor or in the lower parts of the walls. For this reason, they are very difficult to be acquired. Because of their reflectivity, or their inner structure, for example, laser scanner can difficultly be used in this works, as it produces artifacts, which degrade the quality of the point clouds (Godin et al. 2001). The reflections of materials made problems also to record their correct color, as it is strictly depending on the light sources and direction.

A third complex aspect lies in the history of the church. San Marco can be considered a collection of the most important and relevant pieces of art of the entire dominion of Venice in the Mediterranean basin. Very often, capitals, decorations and statues came from different areas and periods and each one has its specific characteristics in terms of form, dimension, position and geometrical complexity. Therefore, it was very difficult to establish a priori a standard rule for acquisition and modelling which could be valid for all elements.

Another factor, which caused significant problems in the survey campaign, is the continuous flow of visitors and restorers inside the church. The basilica is everyday open to the public for touristic visits and religious celebrations and, of course, the presence of people all over the church does not match very well with an intensive and extensive survey campaign. Moreover, San Marco is a big construction site where restorers, carpenters, electricians and marble workers work all the sime, at the same time and in different areas, to maintain the building. This practical problem is obviously typical when dealing with such important buildings. But this difficulty is not related only to the organization of work, but also to the quality itself. The necessity to interrupt a work, both of survey or modelling, and to start it again can change the procedures and practices. It is vital to take this problem into account in the programming phase, as well as in the planning the methodological and technological procedures.

Considering all these aspects, the 3D acquisition of the church has been organized subdividing the whole space into smaller parts, which could have been surveyed independently of one other. This implied the necessity of an adjusted, wellmaterialized and stable topographic network inside the Basilica. It is a classical way to proceed, today a bit snubbed in favor of $3 \mathrm{D}$ real time survey technology, but mandatory when the architecture is extremely large and complex and when the survey must be done in different stages over time. In this way, even two adjacent areas could have been surveyed at different times according to the schedule of activities inside the church. This approach allowed to limit the number of acquisitions, avoiding big overlapping areas (and gaining time for the survey acquisition). Moreover, the topographic network has guaranteed the correct verticality of the reference system and the overall control of accuracy of point cloud registration and of the whole survey process.

The selected technique for data acquisition is digital close range photogrammetry, following the modelling method of dense image matching. This choice is due to two reasons. The first one is the impossibility to use a laser scanner due to the characteristics of the materials; the second is the opportunity to work directly with an image-based approach, optimizing in this way the pipeline using photos firsts for the geometric reconstruction and consequently to build the orthophotos.

To survey and model the whole church, we organized the acquisition in a multiscale way to record the geometry of the architecture space, but also the characteristics of the smallest details, according to the requirements of the representation. For each space, different photogrammetric acquisitions have been computed. The most general one, acquired with $24 \mathrm{~mm}$ (or $35 \mathrm{~mm}$ ) lenses, allowed to obtain the overall geometry of the space, enough to assure an object reconstruction and measurement extraction at a minimum representation scale of 1:50. Another acquisition was usually done with a $85 \mathrm{~mm}$ lens in order to get more details about molding and statue, to extract reliable 3D profiles and mesh models. In some cases, according to the distance between the object to be surveyed and the position of cameras, also $200 \mathrm{~mm}$ lenses have been used to have very high-resolution, above all for the orthophotos. In such a process, at the end of the acquisition, for each area we had two or three photogrammetric acquisitions and consequently dense point clouds at different resolution. The most general models have been oriented in the reference system of the Basilica using coded targets acquired by the total station (Leica TS30). On the other side, the high-detailed models have been registered together using point-based alignment inside Agisoft software using the general model as the reference.

The result of the survey stage has been a very large number of point clouds with different resolution, registered in a single reference system (Figure 1). This multiscale photogrammetric approach gives the possibility of switching on or off the point clouds according to the necessity of inspecting a detailed element or the entire space. This can be done inside the modelling software or in external point cloud viewer.

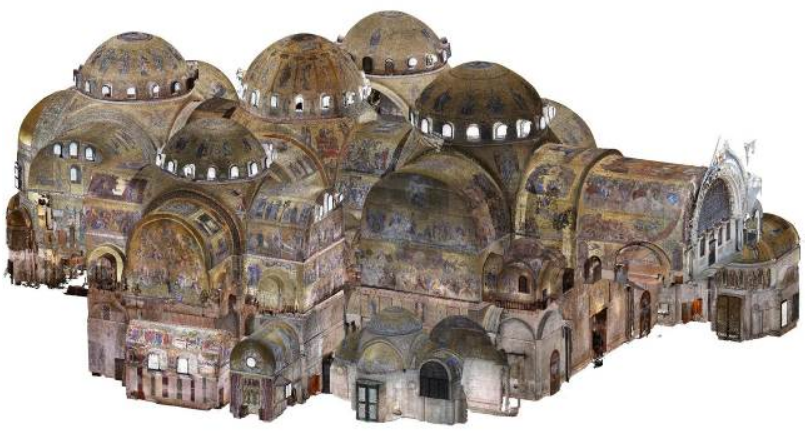

Figure 1: Union of all point clouds of the interior of the Basilica. The whole model is composed by 87 Photogrammetric point clouds and circa 9 billion of points.

\section{3D MODELLING OF ARCHITECTURAL ELEMENTS}

Some difficulties encountered in the survey stage, affected also the modelling process. It has not been possible to define families of repetitive objects inside the church, because of their different origins. Anyway, this problem reflects one of the difficulties of the BIM approach to Cultural Heritage: it is not always possible to identify common constructive rules for architectural elements. It depends on the complexity of the architecture and on its details, on the goal of the BIM, and on the relevance of the architecture (and the corresponding economic effort). For this reason, the choice to build the 3D model using Rhinoceros in place of more common commercial BIM authoring software (such as Revit or ArchiCad) is fully 
justified by the possibility of obtaining very complex realitybased models. The choice of NURBS modelling, moreover, guarantees low file dimension (a relevant condition for big and complex objects), the possibility to integrate in the model also free-form objects and to guarantee the ability to modify in a simple way the object now, during the modelling phase, but also in the future, for the next transformations. The subdivision of the work into smallest parts and the characteristic of continuity of the surfaces is not a simple task of the modelling stage from a practical and organizational point of view; the same issue arises from a conceptual point of view, in order to maintain the topological correctness and accuracy in the final geometry. This last aspect is also more complex wherever the edges are not straight, but smoothed. A solution has been found in the "work session" feature of Rhinoceros, which allows sharing the work between all participants to the working group. It is an index file, which manages a series of 3D models and allows multiple users to navigate and work on them like it was a unique one. In this way, the modellers can work on different areas and, at the same time, share their models.

The 3D modelling approach, in a NURBS environment, is quite well known and it corresponds to the retopology of the object using NURBS surfaces in order to simplify and lighten it, maintaining the correct construction features. It is based on the extraction of the generating features (corners edges or sections) of the object from the point cloud. This manual operation that can be done directly into Rhinoceros using the photogrammetric point cloud as reference and using some supporting plugin as for example Pointools for Rhino ${ }^{2}$ or Arena4D able to visualize huge point cloud and to extract new views and slices from the point-clouds directly in Rhinoceros environment. This approach has been used for several elements such as walls, mouldings and decorations. In each case, the number and position of sections are not fixed, but they have to be decided according to the characteristics and state of the object itself. If a moulding has some deteriorated parts, in that position we need more sections in order to describe that effects.
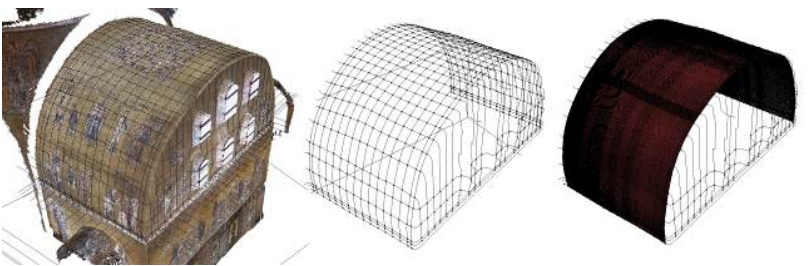

Figure 2: Vault modelling from slices extraction from point cloud (left), network of curves (centre), 3D surface (right)

A very similar approach was used to build vaults and domes (Figure 2). The curves, in both longitudinal and transverse directions, were extracted by the point cloud to create a virtual network of slices, where each knot must belong to both direction sections. Also in this way, quite far from the automation, the distance between subsequent slices must be set according to the deformation and complexity of the vault.

On the contrary, individual sculptural elements that do not interact with the structure (such as capitals and statues) have been modelled by mesh models and computed directly from the point clouds. Even if the dimensions in terms of memory occupation grows a lot, this method allows to keep the details obtained from the survey.

\section{THE PROCESS FOR THE ORTHOPHOTO}

${ }^{2}$ We used this plug-in in the project but no longer available on the market.
The process for the creation of the orthophoto runs across the survey and modelling stage because of the need of a detailed 3D model (more in general a Digital Surface Model) to compute the projection. As the requirements were for highresolution orthophotos, we had to use some expedients to guarantee the best results.

\subsection{The image acquisition.}

The orthophotos should guarantee the 1:1 plotting scale. That means guaranteeing, at least, a pixel size of $0.5 \mathrm{~mm}$ in order to distinguish the junctions between tesserae that is about 1-2 mm. The acquisition was conducted assuring an initial Ground Sample Distance (GSD) of about $0.2 \mathrm{~mm}$, a safety threshold that will guarantee the final desired resolution.

In the survey stage, as described before, the image acquisition has been done following the rules for a correct 3D reconstruction, in terms of distance from the object (to reach the predefined GSD, according to focal length), overlap among images and geometry of acquisition. The images for the orthophoto usually were acquired with tele lens (85mm or 200 $\mathrm{mm}$ ) in order to have a very high resolution.

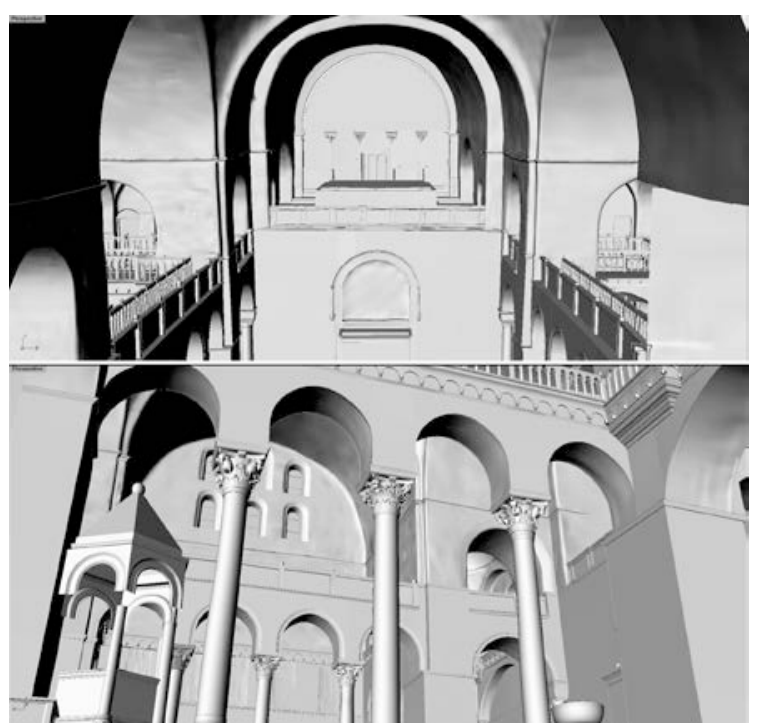

Figure 3: various views of the 3D model.

A big problem inside the Basilica is related to the light conditions. In fact, there were many lighting sources in different positions, with different orientation, intensities and colour, so it is very difficult to have a well distributed light and correctly set the camera for a correct colour registration and to assure a final homogenous exposure. Natural light coming from the windows and existing illumination could not be eliminated, but had to be be reduced: the use of external extra illumination was mandatory. We used three Airstar lighting balloons: 2 Sirocco 2500W HMI Hot Restrike to be used coupled in large spaces and one little Sirocco 575W usable in narrow spaces or for decorations. The lamps produced a very homogenous light $\left(280^{\circ}\right)$, with the known light temperature of $5600{ }^{\circ} \mathrm{K}$. They could be attached to long and stable poles up to the height of 8 $\mathrm{m}$ in order to light up also the upper parts of the Basilica or to "block" the inflowing natural light. Small led spotlights used to correct possible reflections or shadows in defined area as in Figure 3.

All the images were acquired in RAW format in order to assure the maximum possibility of editing during the photo postprocessing (mainly white balance and colour correction) and taken using the tripod in order to avoid micro-movement or 
blurry images and to assure the maximum micro contrast.

After the acquisition, all the images were processed in Agisoft Photoscan, divided by group in function of the focal lenses that had been used, to orientate them and to produce the matched dense point model. In the first stage a few target and a discrete number of architectonic GCPs were used to support the correct image alignment and the geo-referencing of the data.

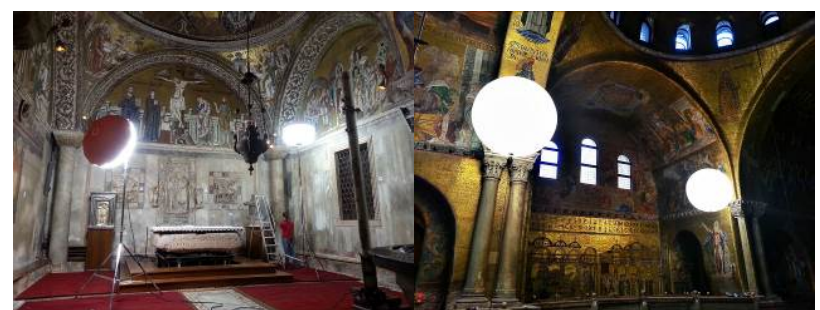

Figure 4: Lighting systems during the acquisition stage: detail of the balloons.

\subsection{The modelling phase.}

The matching was computed normally in high-mode, that means reducing the original resolution by a factor 4 ; image redundancy and multi-image overlapping guarantee the final density of the point cloud of about $5 \mathrm{~mm}$.

At this point the meshing part, the creation of the mesh surface inside Agisoft Photoscan was skipped. As described before, the point clouds were used to create the final 3D model, by direct modelling approach inside Rhinoceros. This because:

1. The model must be simplified

2. Different model should be correctly joined and integrated together

3. Mesh surfaces can be hardly modified

4. Mesh sculpting or modelling is difficult in order to guarantee a reality based accurate reconstruction

5. The smart segmentation of the model is difficult on mesh model

6. Complete hidden or shadow areas is a challenging task using mesh

7. Mesh model is far away conceptually from any architectonic representation: difficult to be understood and managed by professional.

8. Classical drawings or measurement cannot be extracted without a time consuming work.

All these motivations are the reasons why the modelling stage was conducted inside an external software and why we pass from a raw mesh model to a complete, segmented even simplified NURBS model.

\subsection{The use of the final model.}

At this stage, the final 3D model followed two separated paths. The first one, original NURBS model, is used inside Rhinoceros to extract automatically 2D information, plans, section and elevations. The second one was exported as mesh surfaces and re-imported into Agisoft Photoscan, as DSM for the creation of orthophotos and to be textured as visualization model used inside the web part of the BIM3DSG system. Even if the model is simplified, it is accurate enough for both goals, corners and the main features are in the correct position. The simplification concerns only a general smoothness of the surfaces, the elimination of some irregularities due to external objects as, for example, fixed furniture or power lines and boxes.

\subsection{The orthophoto creation.}

Before proceeding with the orthophoto, a selection of images was picked up in order to use only the best ones. All the images with a strong different colour or acquired from a bad convergent angle were rejected, in the attempt to keep only the ones which could be considered orthogonal to the surface. This was possible because of the redundant number of images required for $3 \mathrm{D}$ reconstruction.

At the end of the process, the orthophoto was calculated with a GSD of $0.5 \mathrm{~mm}$ using the best architectural planes, identified by markers on the surface.

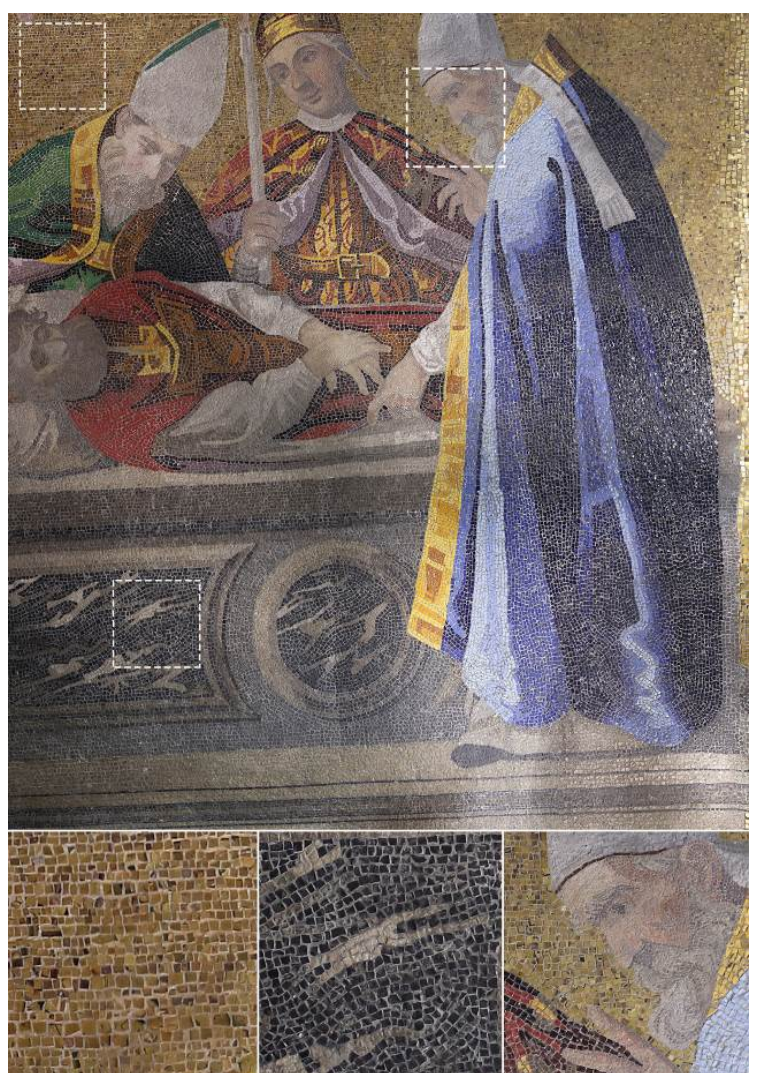

Figure 5: Orthophoto with some details that show different tessera sizes and disposition in the same mosaic.

In order to allow an easy use of the orthophotos, they have been organized in small areas according to the restoration necessity, the iconography of the content and the characteristics of the architectural element. This is the case of the main arches: their intrados is decorated with mosaics and so it was necessary to unwrap it to obtain a planar representation. A different intervention is necessary for the domes, where it is not possible to unwrap correctly the surface into a single plane. The choice is taken case by case in accordance with the Procuratoria di San Marco and selecting from time to time the part interested by maintenance and choosing the right best projection plane.

In some case, it has been necessary to edit the orthophoto to allow a better readability, by manually choosing in Agisoft Photoscan different projected images.

\subsection{The texture creation.}

All the mesh models were textured inside Agisoft Photoscan using one texture atlas. The dimension of the object and resolution of the texture atlas are calculated from time to time in order to guarantee a minimum texture resolution of $1 \mathrm{~mm}$ and considering that the system cannot visualize texture bigger than 4096x4096 for memory consumption requirement. Moreover, at 
the moment the information system and the web viewer are not able to visualize multi-texture object.

This operation is metrically precise because the texture is calculated photogrammetrically. It very useful not only to represent the 3D model in a more "realistic" way and to navigate inside the model as a living $3 \mathrm{D}$ catalogue of mosaics but also, in particular, to take measurements directly in 3D using texture points as reference.

\section{THE BIM SYSTEM}

In order to manage, store and share data (3D model and orthophotos) the idea was to use a BIM-like system meant as an instrument able to enhance the potential of using the 3D digital model of the Basilica during the extraordinary and ordinary restoration and maintenance activity. The BIM System should be the base to extract 2D and 3D measurements, but also the 3D repository of data and information connected to the model and the spatial catalogue for all high-resolution orthoimages created during the project.

With this in mind, the general objective was to create an ad hoc information system characterized by:

1. Extreme user-friendliness so that it can be used by even the most unexperienced 3D operators internally and externally to the Procuratoria di San Marco;

2. High degree of interoperability between operators who can work on real-time activities on the same object in the same moment from different workstations;

3. Possibility of cooperation (even with extended audience): each registered actor can upload or simply visualize data, modify and update information in a perfect "wiki" style.

\subsection{The architecture of the system.}

The BIM system adopted here is an ad hoc adapted version of BIM3DSG system, already presented in Rechichi et al., 2016 and, in particular, it is an updated version of the WebBIMDuomo (Fassi et al. 2012).

The philosophy of the system is to have at disposal a unique information system created specifically for Cultural Heritage, which is therefore suited to the most diverse realities. It is important to consider that architectural structures have different needs, functions and problems, operators, management and maintenance modes. For this reasons the implemented system should not be a rigid pre-packaged, but rather a dynamic system that can be applied to different study cases. It is then necessary to rely on a diversified database and on a different modelling philosophy depending on the multiple needs, on the characteristic of the structure but also on the methodological and operational choices adopted by the different operators. To achieve this goal, the main idea was to create a BIM system in which, following the simplest and most general definition, the digital 3D model is associated with various types of information that can change in relation to the different case of study.

BIM3DSG is divided in three parts:

1. The "modelling part" that relies on existing modelling software (Rhinoceros), by giving them the onerous task of creating and updating the 3D model.

2. The "user parts" developed on web platform that allows the navigation of the model and the user interaction with the information both in reading and writing mode. It focuses in particular on the part of the use of digital model as a data catalyst. It is a visual data entry application that works online and allows immediate use and easy learning of the 3D model as a catalogue of information.

3. The central database that contains all the data and information.

\subsection{The dedicated system}

For the Basilica di San Marco the original architecture of the system was used (Fassi et al., 2015). Some ad hoc implementations were developed in order to:

9. visualize the high-resolution textured models on the web and consequently to manage the import/export of the texture;

10. visualize and navigate high-resolution orthophoto;

11. manage and synchronize the NURBS model created in Rhinoceros with the textured mesh coming from the successive passage in Agisoft Photoscan.

As explained before, the NURBS model was created in Rhinoceros. It should have been a reality-based model segmented in every constructive element, in this case geometrically (e.g. columns divided from capital and bases) but also iconographically (e.g. different mosaic figures on the walls) and structured spatially and/or logically in four categories. Each parts were exported inside the system as single *.3dm file in order to be downloaded and reimported in a later time inside Rhinoceros; both processes are automatically handled by a BIM3DSG plug-in for Rhinoceros.

In the original system during this export operation the models was also exported in *.JSON format for the online visualization: this feature cannot be applied to this case of study as it is, because Rhinoceros cannot fully handle the texture on NURBS model, showing some important issues when generating the visualization mesh for texturized NURBS. For this reason, the export was split into two-separated procedure, developing a second one specifically designed for the web visualization: it can be run either with BIM3DSG Rhino plugin or through a standalone software and automatically generate the needed JSON for all LoD for each object starting from the texturized mesh models. The existent export procedure was revised with the capability to export only the *.3dm file useful for future update of the model. The two models (3dm and JSON) are connected together and related to the single object but can be modified independently. The eventual synchronization of the two part should be obviously done manually.
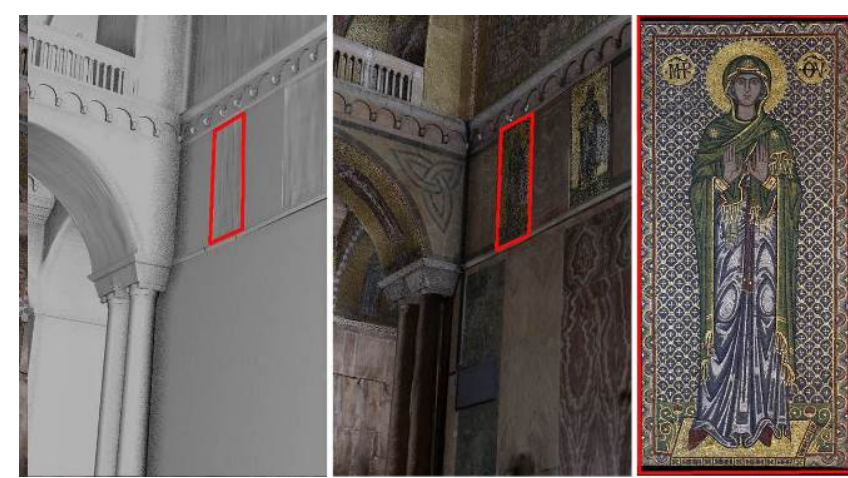

Figure 6: location of orthophoto from 3D NURBS model and textured model

6.2.1 The texture function. Texture are applied loading the UV texture map (Mullen, 2009), using the UV coordinates that are saved inside the JSON files. The texture files can be JPEG or PNG and are stored inside the database. There is an ad hoc 
cache for texture, always using the web browser persistent storage, such as for models, that allows to have almost instantaneous loading time after the first time, even on low speed internet connection.

The max resolution supported for the texture is $4096 \times 4096$ pixel, thus if the texture provided by the user is at a higher pixel size, it is automatically reduced during the export process. For the LoD of the texture, the max resolution texture is assigned to the LoD0, while for the lower LoD the image is resized using the pixel resize technique, halving the pixel size at each step. When the user uploads object into the web browser, he can choose the LoD of the texture independently from the LoD of the model, in order to adapt to the graphic resource and memory which are available on the current device, and to the number of uploaded objects. Moreover, the user can change, on the fly, the LoD of each uploaded object, without the need to reload the entire scene; this feature is very useful to view some highquality texture after loading the entire scene at lower LoD on low level device. BIM3DSG has only the capability to enable or disable the web texture visualization on the fly, for analysis purposes.

6.2.2 The orthophoto functions. The BIM3DSG supports the management and the visualization for both extremely highresolution orthophotos and/or image in general. The choice was to integrate an existent open source software inside BIM3DSG system.

Extremely high-resolution images and orthophotos mean that there is no limit in resolution and image dimension. They can be uploaded in chunk to the BIM3DSG server using Ajax File Uploader and then reassembled remotely with php. When the upload is completed, the system automatically processes the tiff image in order to create a compressed Tiled Pyramidal TIFF. It is a standard TIFF extension, supported by most image processing applications, and is simply a tiled multi-page TIFF image, with each resolution stored as a separate layer within the TIFF. The conversion is done using VIPS. It is a free image processing system executed on the server through php. After the conversion, the resulting Tiled Pyramidal TIFF is automatically moved in the dedicated folder (one for each BIM3DSG project) and renamed using a specific convention that allows associating each file to the specific connected object.

For the visualization, BIM3DSG integrate IIPMooViewer, a lightweight high performance HTML5-based JavaScript. It allows high-resolution image streaming and zooming. It is client compatible with almost all web browsers and mobile browsers, with the ability to zoom, navigate and rotate the images. IIPMooViewer is open source and it is specifically designed to work with the IIPImage server. It is an open source Fast CGI module written in $\mathrm{C}++$ that is designed to be embedded within a host web server such as Apache, used by BIM3DSG system.

6.2.3 The orthophoto functions-client side. The orthophoto functions are completely available to the users. In this context, we consider as a user both the operator who computes and uploads the orthophoto, and the operator who downloads them for different purposes.

The first operator, who creates the orthophoto, does not need to access the core of the system, as can use an easy procedure to upload the images (and automatically produced the Tiled Pyramidal Tiffs described before).

The side of the final operator is very user-friendly too (Figure 7). It guarantees that it is not necessary a deep knowledge of the system to manage orthophotos. By exploring the model in the web viewer, the user can select one element and open a menu of linked images. The possibility to navigate in the textured model fosters the operation of orthophoto selections and does not require the use of lists or specific codes to find the desired orthophoto. When the user selects the image, a navigation system appears and it is possible to zoom the image to see all details. The presence of the navigation tool and the representation of the scale on the bottom right simplify the exploration and knowledge of the orthophoto.

\section{CONCLUSIONS}

In the paper, it is briefly described the process which has been designed to create a smart informative system useful for the maintenance of the mosaics of the Basilica di San Marco in Venice. The main issue during the project are explained and critically described as they were resolved.

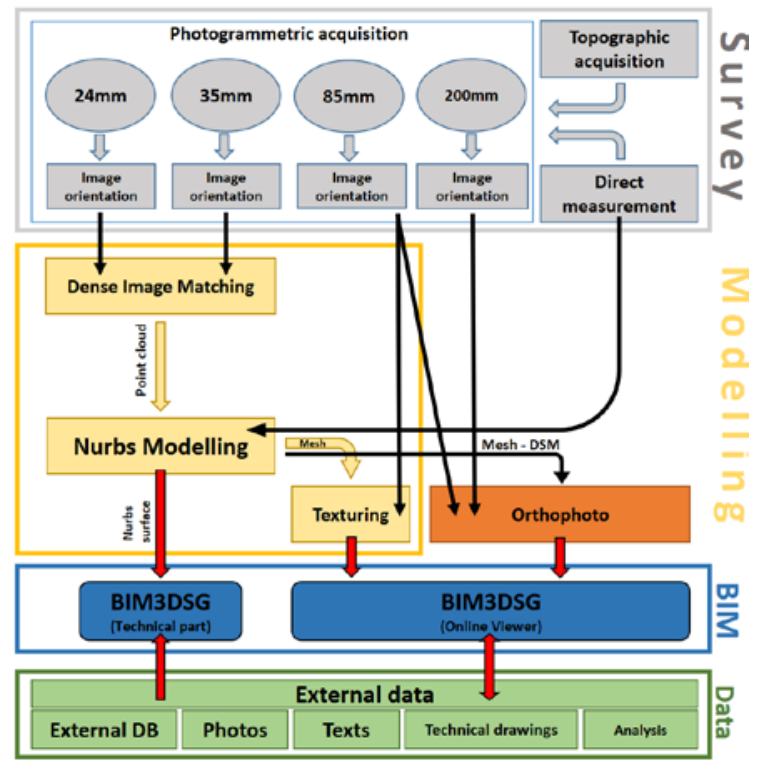

Figure 7: Pipeline of the project from the survey part, through the modelling part, to the orthophoto creation and the use of BIM3DSG system as catalyst of information.

The main problematic in the survey phase was the $3 D$ acquisition of huge and high spaces characterized by reflective materials and bad environmental light condition. This and the final goal of the project led to the choice of the Close Range Photogrammetry as the right method to build an accurate 3D model of the Basilica and to have at disposal extremely high resolution images for 1:1 orthophoto creation. A multiresolution and segmented acquisition was necessary.

The modelling phase was surely the most time-consuming and challenging part. The 3D model should first of all play the role of a catalogue of the mosaics of the Basilica. For this reason, the modelling phase takes care only on external surfaces ignoring at this stage the volumes. The second purpose is the automatic extraction of sections and elevation at 1:50/1:100 representation scale. This means that the modelling phase should ensure great accuracy $(1-2 \mathrm{~cm})$. Rhinoceros was chosen as the best software solution in order to extract a reality based model from point cloud generated by photogrammetric image matching. In order to create the orthoimages, an accurate and dense DSM of the object is necessary. Even if the natural choice would have been to continue with the photogrammetric pipeline inside Agisoft Photoscan, the right choice was to use the model created in Rhinoceros simplifying the geometry and completing the model respecting the topological relation between elements. The NURBS model exported as DSM for the 
orthophoto projection and for the texturing is accurate enough for required representation scale. This assures that the same model is used in Rhino as NURBS to extract automatically 2D views and the web in the BIM system viewer.
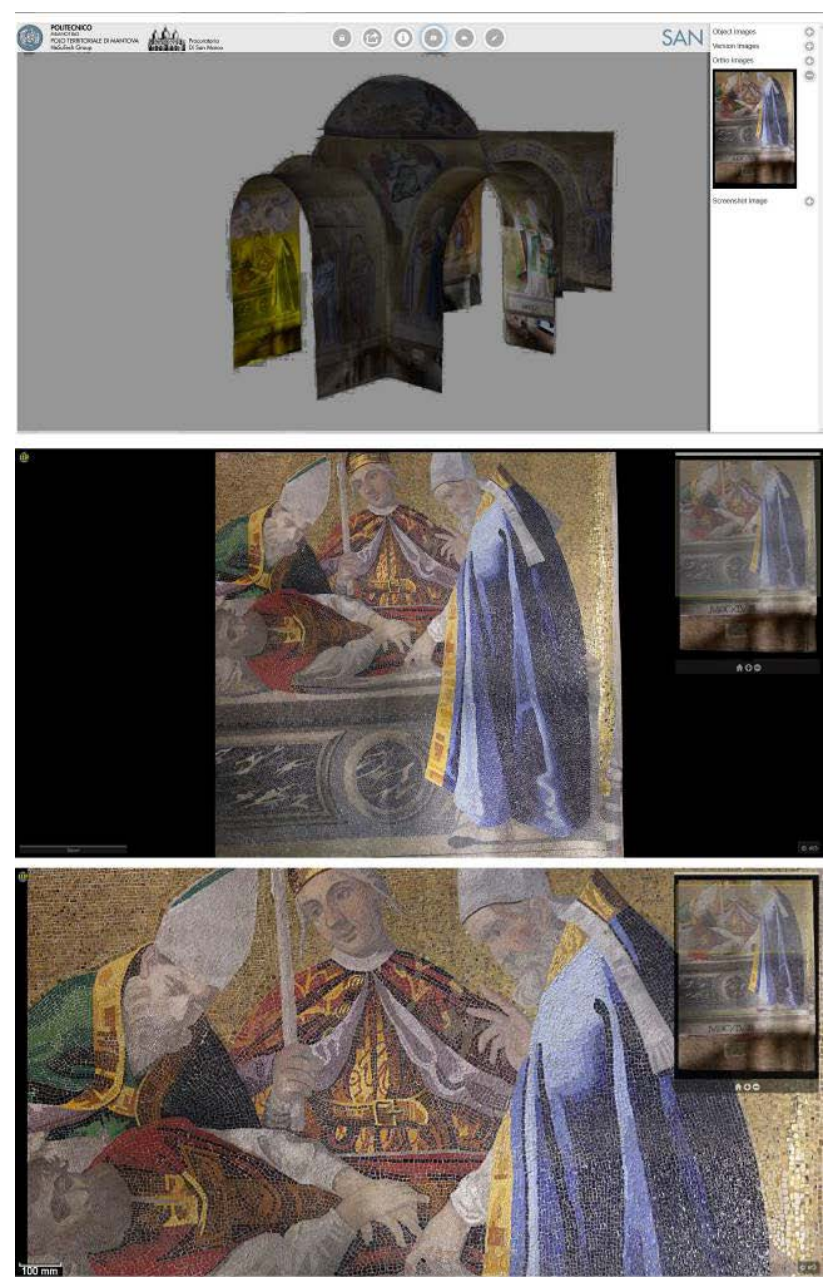

Figure 8: The orthophoto fruition by the user (from the top): i) selection of the element and choice of the orthophoto to be viewed, ii)orthophoto visualization with navigation tools, iii) visualization of details of the orthophoto at the maximum zoom, with scale bar and navigation tool.

3D surface model, 3D textured mesh and orthoimages are stored and interconnected inside the BIM3Dsystem created ad hoc to manage these data. The system allows the navigation of the textured 3D model, the measurement of simple distancea and coordinates, the possibility to add or update information, notes and external files in the information system. To each object is connected one or more high-resolution orthophoto that can be visualized at full resolution in a separated webpage. This process, implemented for orthophotos management, works for each kind of image, so it becomes a way to collect many types of data connected with the single element (historical images, the state of conservation, cracks description, mapping drawings etc.). The system is useful as data repository, to collect and share restoration information among the different user of Procuratoria di San Marco.

Future works are the development of smart web tools in order to work directly on the orthophoto inside the web, in order to map activities or punctual data entry. A second future task could be to dynamically connect $2 \mathrm{D}$ orthophoto with the 3D model allowing the extraction of self-built orthoimages, after independently choosing the area of interest and projection plane. This could disengage the system from the rigid constraint due to the pre-segmentation of the data made in the modelling phase and consequently in the creation of the orthophoto.

\section{ACKNOWLEDGEMENTS}

This work was supported by Procuratoria di San Marco. Special thanks to the ex-Proto Arch. Ettore Vio, who promote the research project and Arch. Mario Piana, current Proto, for his support and availability.

\section{REFERENCES}

Fai S., Graham K., Duckworth T., Wood N., Attar R., 2011. Building Information Modeling and Heritage Documentation, CIPA 2011 Conference Proceedings: XXIIIrd International CIPA Symposium.

Fassi F., Achille C., Mandelli A., Rechichi F., Parri S., 2015: A new idea of BIM system for visualization, sharing and using huge complex 3D models for facility management. The International Archives of the Photogrammetry, Remote Sensing and Spatial Information Sciences, Avila, Spain, Volume XL5/W4, pp. 359-366.

Fassi, F., Campanella, C.: From daguerreotypes to digital automatic photogrammetry. Applications and limits for the built heritage project, Int. Arch. Photogramm. Remote Sens. Spatial Inf. Sci., XLII-5/W1, 313-319, doi:10.5194/isprs-archivesXLII-5-W1-313-2017, 2017.

Fassi F., Parri S. (2012). Complex Architecture in 3D: From Survey to Web. International Journal of Heritage in the Digital Era, vol. 1, p. 379-398, ISSN: 2047-4970

Fregonese L., Monti C., Monti G., Taffurelli L., 2006: The St. Mark's Basilica pavement. The digital orthophoto 3d realization to the real scale 1:1 for the modelling and the conservative restoration in Innovations in 3D Geo Information Systems, First International Workshop on 3D Geoinformation, 7-8 August, 2006, Kuala Lumpur, Malaysia.

Fregonese, L., Taffurelli, L., Adami, A., Chiarini, S., Cremonesi, S., Helder, J., and Spezzoni, A., 2017. Survey and modelling for the BIM of Basilica of San Marco in Venice, Int. Arch. Photogramm. Remote Sens. Spatial Inf. Sci., XLII-2/W3, 303-310, doi:10.5194/isprs-archives-XLII-2-W3-303-2017.

Godin, G., Rioux, M., Levoy, M., Cournoyer, L., \& Blais, F. , 2001. Marble Surfaces. October 2001.

Mullen, T (2009). Mastering Blender. 1st ed. Indianapolis, Indiana: Wiley Publishing, Inc. ISBN 9780470496848

Rechichi, F., Mandelli, A., Achille, C., and Fassi, F, 2016. Sharing high-resolution models and information on web: the web module of BIM3DSG system, Int. Arch. Photogramm. Remote Sens. Spatial Inf. Sci., XLI-B5, 703-710, doi:10.5194/isprs-archives-XLI-B5-703-2016

http://www.sanwebe.com/2012/06/ajax-file-upload-with-phpand-jquery

http://www.vips.ecs.soton.ac.uk/index.php?title=VIPS

http://iipimage.sourceforge.net/documentation/ 